\title{
Video Diagnostic for W7-X Stellarator
}

\author{
J. Sárközi ${ }^{\mathrm{a}}$, K. Grosser ${ }^{\mathrm{b}}$, G. Kocsis ${ }^{\mathrm{a}}$, R. König ${ }^{\mathrm{b}}, \mathrm{U}$. Neuner ${ }^{\mathrm{b}}$, Á. Molnár ${ }^{\mathrm{a}}$, \\ G. Petravich ${ }^{\mathrm{a}}$, G. Por ${ }^{\mathrm{c}}$, G. Porempovics ${ }^{\mathrm{d}}$, S. Récsei ${ }^{\mathrm{a}}, \mathrm{V}$. Szabód ${ }^{\mathrm{d}}$, A. Szappanos ${ }^{\mathrm{a}}$, \\ S. Zoletnik
}

\author{
${ }^{a}$ KFKI-Research Institute for Particle and Nuclear Physics, EURATOM Association, H-1121 Budapest, Konkoly \\ Thege 29-33, Hungary \\ ${ }^{b}$ Max-Planck-Institut für Plasmaphysik, Teilinstitut Greifswald, Wendelsteinstr. 1, D-17491 Greifswald, Germany \\ ${ }^{c}$ Budapest University of Technology and Economics - Institute of Nuclear Techniques, P.O. Box 91, H-1521 \\ Budapest, Hungary \\ ${ }^{d}$ Budapest University of Technology and Economics - Department of Applied Mechanics, EURATOM Association, \\ P.O. Box 91, H-1521 Budapest, Hungary
}

\begin{abstract}
The video diagnostics for W7-X - which is under development - is devoted to observe plasma and first wall elements during operation, to warn in case of hot spots and dangerous heat load and to give information about the plasma size, position, edge structure, the geometry and location of magnetic islands and distribution of impurities. The video diagnostics will be mounted on the tangential AEQ-ports of the torus that are not straight and have about $2 \mathrm{~m}$ length and a typical diameter of $0.1 \mathrm{~m}$ which makes its realization more difficult. The geometry of the 10 tangential views of the AEQ-ports allows giving an almost complete overview of the vessel interior making this diagnostic indispensable for the machine operation. Different concepts of the diagnostics were investigated and finally the following design was selected. As a large heat load is expected on the optical window located at the plasma-facing end of the AEQ-port, the port window is protected by a cooled pinhole. An uncooled shutter located behind the pinhole can be closed to prevent window contamination during vessel conditioning discharges (glow discharge cleaning) and from inter-pulse deposition of soft a-C:H layers. The imaging optics and the detection sensor are located behind the port window in the port tube, which will be under atmospheric pressure. To detect the visible radiation distribution a new camera system called Event Detection Intelligent Camera (EDICAM) is under development. The system is divided into three major separated components. The Sensor Module contains only the selected CMOS sensor, the analog digital converters and the minimal electronics necessary for the communication with the subsequent camera system module called Image Processing and Control Unit (IPCU). Its simple structure makes the Sensor Module suitable to operate despite being exposed to ionizing (neutron, $\gamma$-) radiation. The IPCU, which can be located far from the Sensor Module and therefore far from the plasma, is designed to perform real time evaluation of the images detecting predefined events, managing the sensor read-out and the input triggers and producing the output triggers generated by the detected events. The IPCU can also be used to reduce the amount of the stored data. A Standard 10 Gigabit Ethernet fiber optics connection connects the IPCU module to the PC with GigEVision communication protocol.
\end{abstract}

Keywords: Stellarator, Video camera, Plasma diagnostics, Event recognition.

PACS: $52.55 . \mathrm{Hc}, 52.70 .-\mathrm{m}, 01.50 . \mathrm{ff}$

\section{INTRODUCTION}

The W7-X Stellarator requires high-speed video diagnostics in order to monitor the plasma and first wall elements during operation. The video diagnostics must be able to warn in case of hot spots and dangerous heat loads. It must also be able to give information about the plasma size, position, edge structure, the geometry and location of magnetic islands and distribution of impurities. The long, approximately 30 minutes continuous operation of the W7-X Stellarator poses special demands both on the mechanics of the video diagnostics and on the video camera system. 


\section{OVERVIEW OF THE MECHANICAL SYSTEM}

To allow an almost complete overview of the vessel interior 10 video camera systems will be installed in the AEQ-ports, providing 10 tangential views. The arrangement of the AEQ-ports is described on the top view of the torus on Figure 1., where the ten AEQ-ports are marked with arrows.

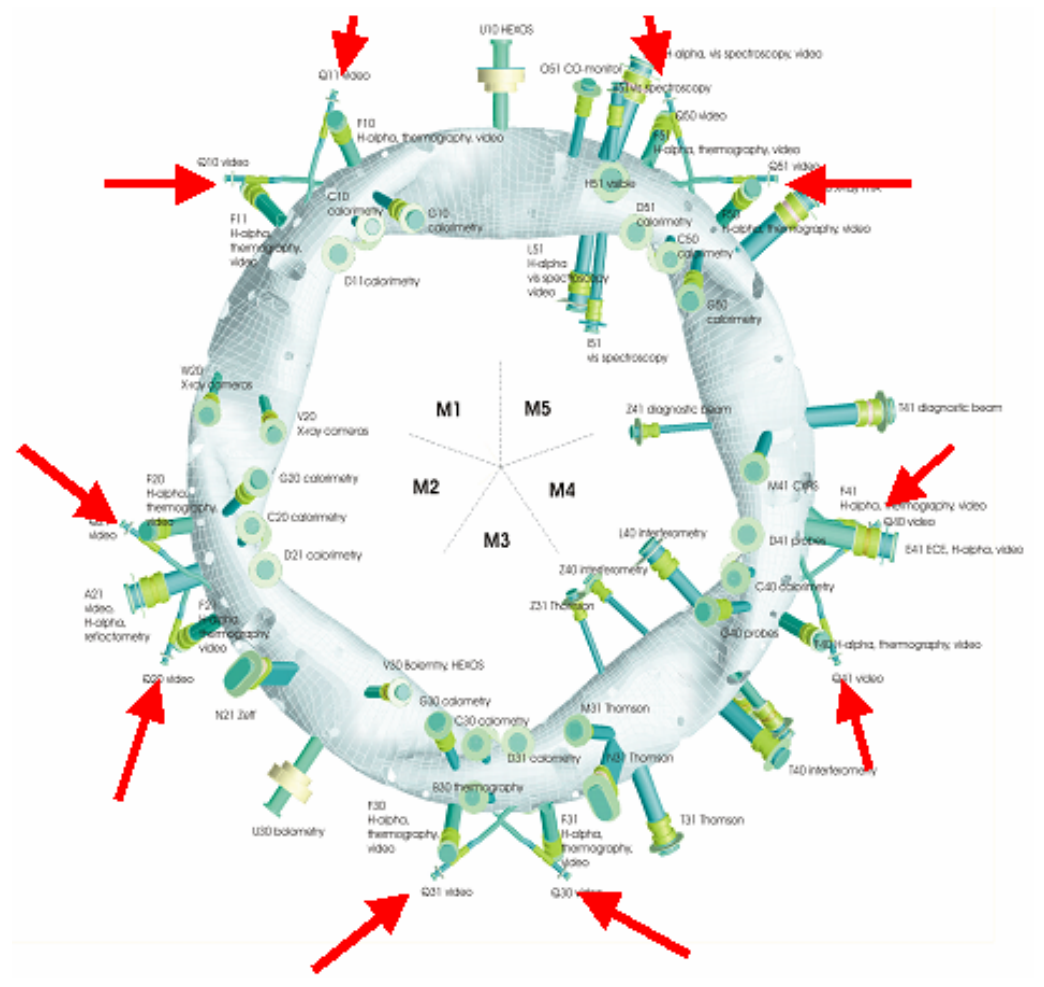

FIGURE 1. Top view of the torus, the ten AEQ-ports are marked with arrows

In contrast to the less than a minute continuous operation of small and medium sized Tokamaks, the W7-X Stellarator can be operated for long time periods of up to 30 minutes. This means, that a large heat load is expected on the optical window located at the plasma-facing end of the AEQ-port. To avoid the overheating of the window, the port window is protected by a water-cooled pinhole. The geometry of the pinhole is matched to the field of view of the imaging optics. Window contamination during glow discharge cleaning of the plasma vessel and from interpulse deposition of soft a-C:H layers will be prevented by closing a small un-cooled shutter, which will be installed directly behind the pinhole, while during discharges the shutter needs to remain open at all times not only because it cannot be cooled due to space limitations but foremost because the video diagnostic is a control diagnostic. The imaging optics and the video camera are located behind the port window in the port tube, which is under atmospheric pressure. The camera-head can be easily removed and reinstalled into the port. For the accurate alignment of the camera-head a docking unit is used. The high ambient temperature in the Q ports requires cooling of the camera-heads. The cooling is performed by compressed air flushing. The cooling air is introduced into the system via the central tube connected to the camera head and leaves the port via the port tube. The mechanical layout of the video camera head can be seen on Figure 2. 


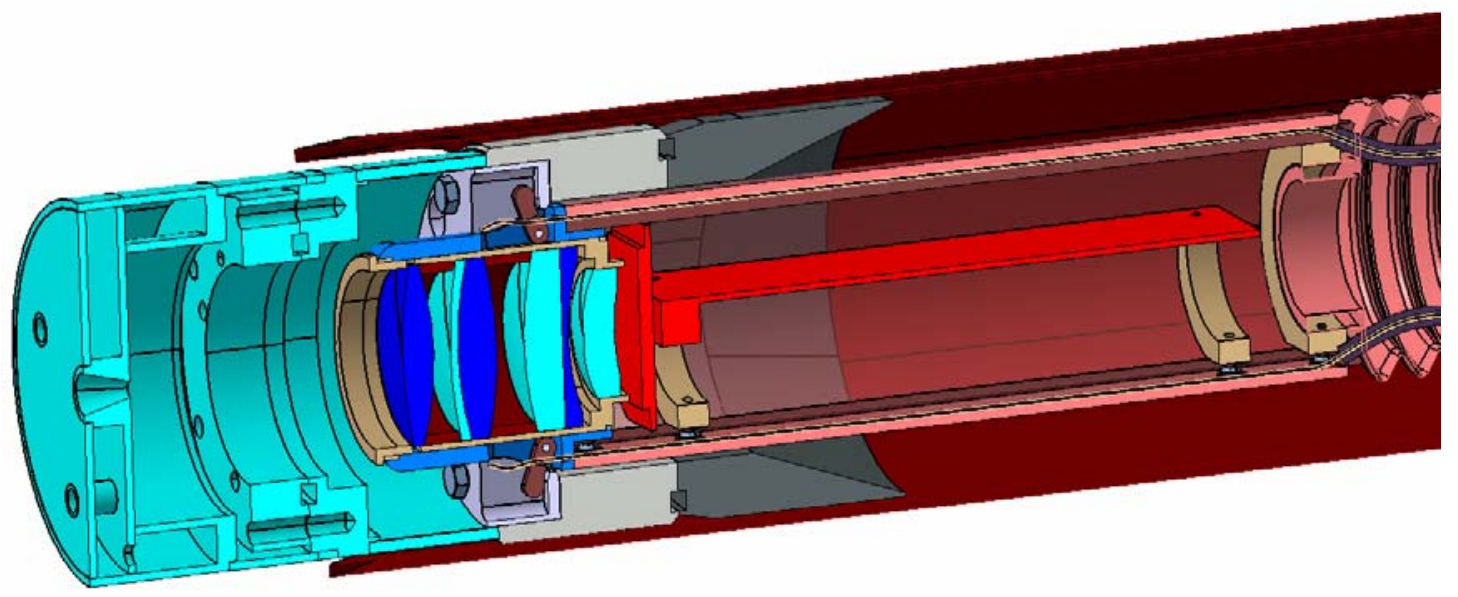

FIGURE 2. Mechanical layout of the video camera head, with a dummy objective and without shutter

\section{OVERVIEW OF THE CAMERA SYSTEM}

To detect the visible radiation distribution in the W7-X Stellarator, a new camera system called Event Detection Intelligent Camera (EDICAM) is under development. Beyond the requirement to be able to operate the video camera under harsh conditions (e.g. neutron and $\gamma$-ray radiation), the long continuous operation of the Stellarator requires special solutions for data handling, as with the highest frame-rate and resolution, 1.43 Terabyte data would be accumulated over a half an hour operation cycle.

The system is divided into three major separated components: The Sensor Module (SM), the Image Processing and Control Unit (IPCU) and the Personal Computer. The three modules are connected with standard 10 Gigabit Ethernet fiber optics communication. The IPCU module is connected to the PC with GigEVision communication protocol. The EDICAM system structure can be seen on Figure 3.

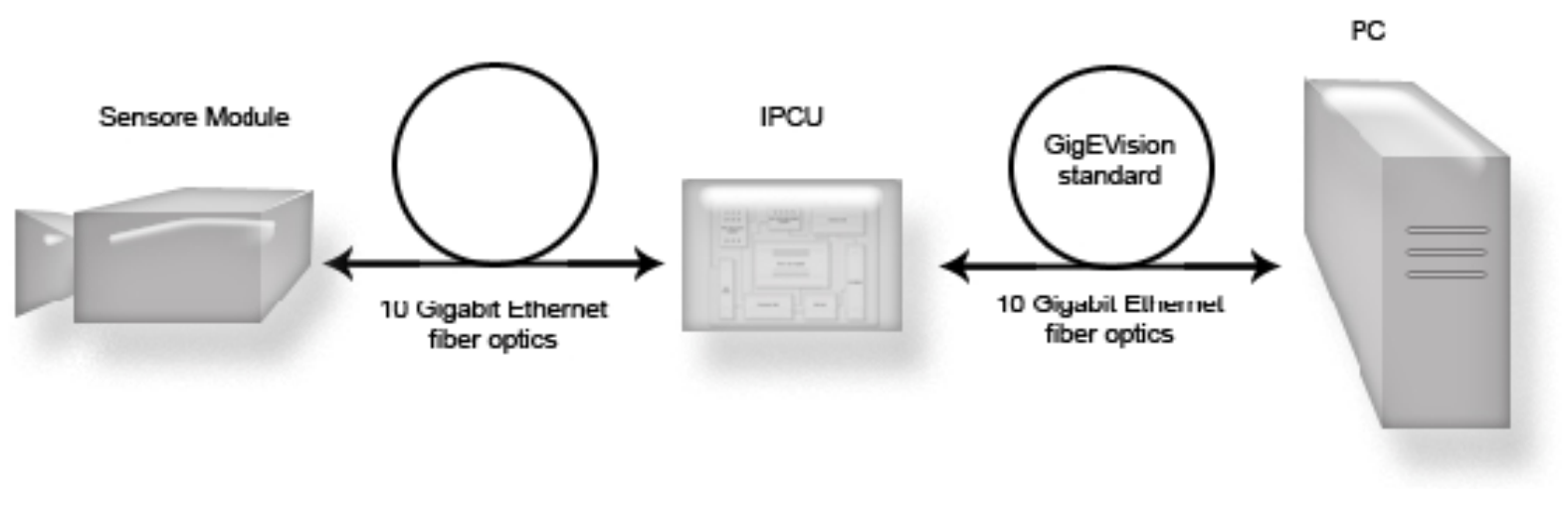

FIGURE 3. EDICAM system structure

The Sensor Module contains only the selected LUPA-1300, 1.3 Megapixel CMOS sensor, the analog to digital converters and a FLASH based FPGA to perform control functions and the communication with the subsequent camera system module. The selected CMOS sensor has 1280x1024 resolution and has several special features, like the windowing capability and non-destructive readout. The simple structure makes the Sensor Module suitable to operate in the harsh environment at the plasma facing front end of a port.

The Image Processing and Control Unit can be located far from the Sensor Module and therefore far from the plasma. All the managing and processing functions are handled by the IPCU: It is designed to perform real time evaluation of the images, detect predefined events, manage the sensor read-out and the input triggers and produce 
the output triggers generated by the detected events. The IPCU can also be used to reduce the amount of the stored data.

The sensor and the IPCU make possible the definition of multiple, arbitrary shaped Region Of Interests (ROI) within the full frame. The ROIs can be read out non-destructively and independently from the full frame. Depending on the number and size of the selected ROIs, the read out speed can be much higher than the read out speed of a full frame. For example, it is possible to read out 444 full frames per second and 100000 frames per second for a single ROI containing 900 pixels. The high level representation of the IPCU event processing function can be seen on Figure 4. Figure 5. shows a picture of the functional prototype video-camera head.

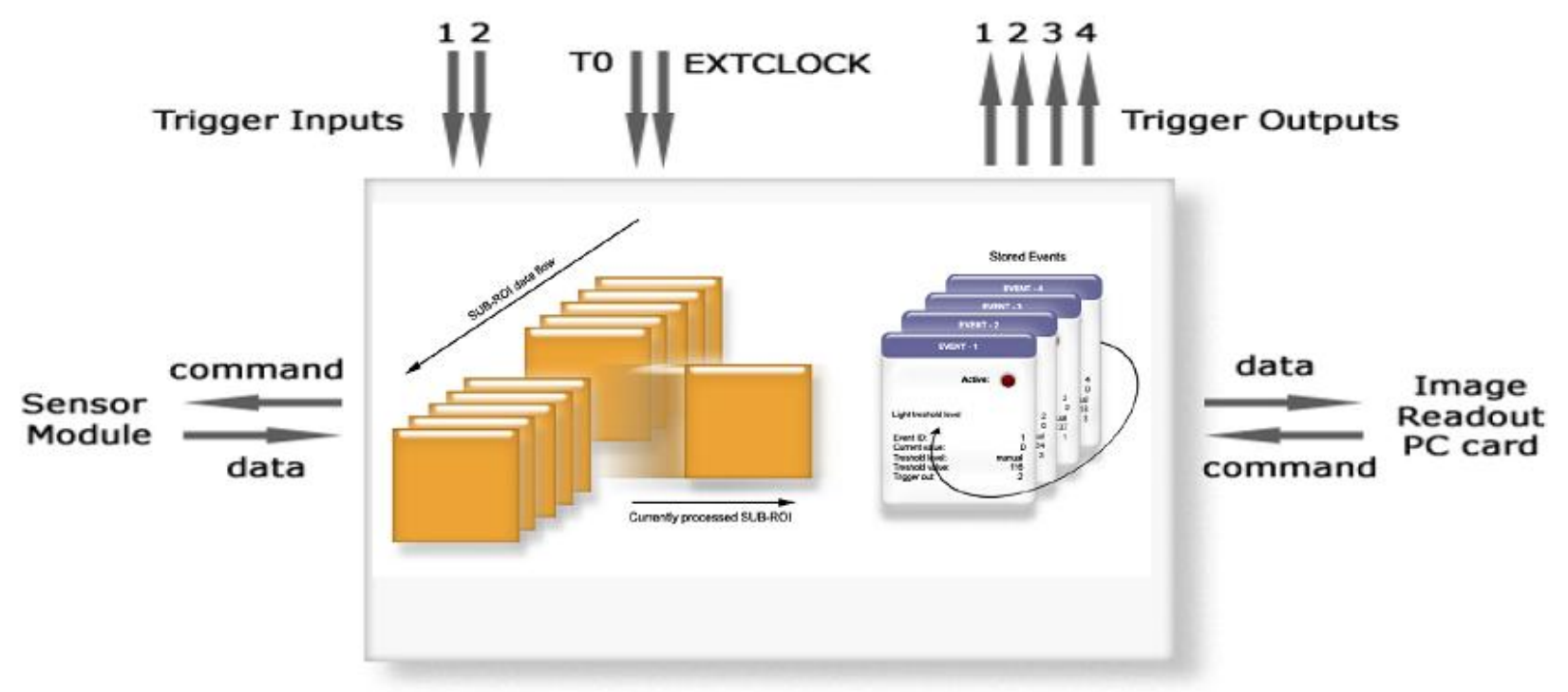

FIGURE 4. The high level representation of the IPCU event processing function.

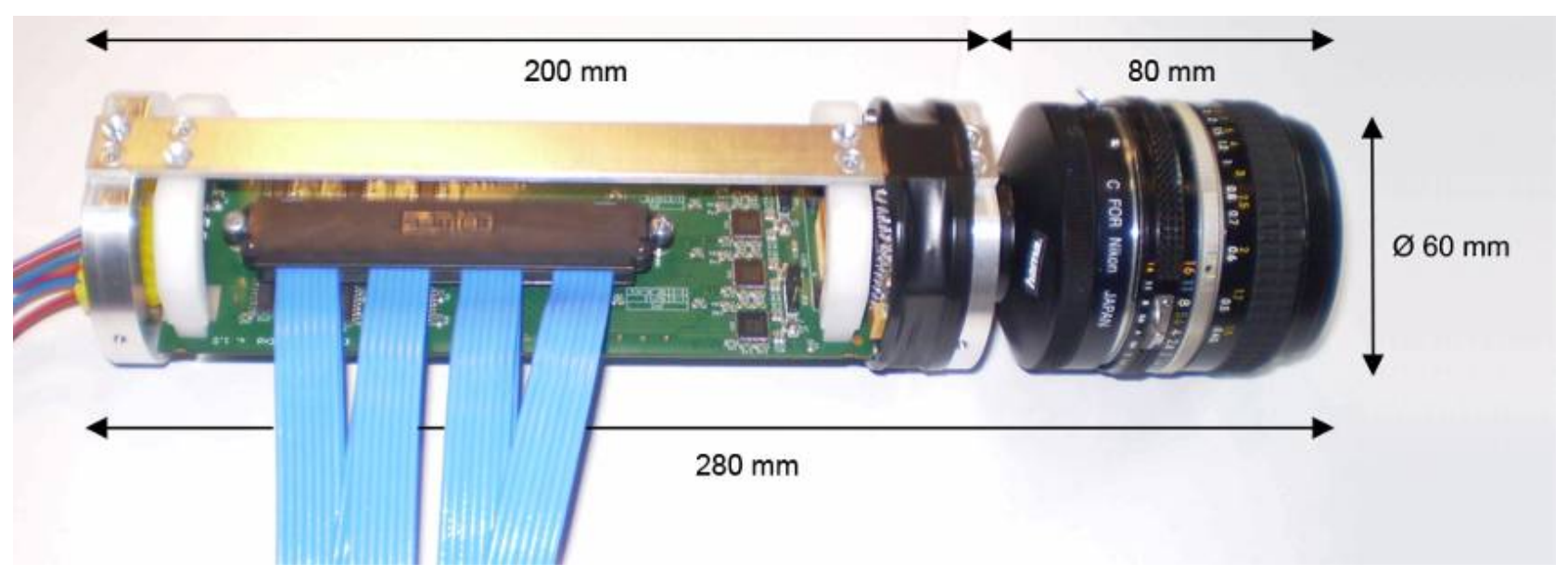

FIGURE 5. The functional prototype video-camera head. 